\title{
Acute right lower limb deep venous thrombosis due to pressure from large adenomyotic uterus-laparoscopic management
}

\author{
Manjula Anagani1 $^{1}$, Prabha Agrawal ${ }^{1 *}$, Rahul Agrawal ${ }^{2}$
}

\begin{abstract}
${ }^{1}$ Department of Obstetrics and Gynecology, Medicover Woman and Child Hospital, Hyderabad, Telangana, India ${ }^{2}$ Department of Internal Medicine, Medicover Woman and Child Hospital, Hyderabad, Telangana, India
\end{abstract}

Received: 29 August 2020

Accepted: 06 October 2020

\author{
*Correspondence: \\ Dr. Prabha Agrawal, \\ E-mail: drprabha1001@yahoo.com
}

Copyright: () the author(s), publisher and licensee Medip Academy. This is an open-access article distributed under the terms of the Creative Commons Attribution Non-Commercial License, which permits unrestricted non-commercial use, distribution, and reproduction in any medium, provided the original work is properly cited.

\begin{abstract}
A 48-year lady came with abnormal uterine bleeding with 22 weeks size adenomyotic uterus compressing on right common iliac, external iliac and femoral vein leading to acute deep venous thrombosis (DVT). She had history of severe anaemia secondary to menorrhagia with multiple blood transfusions and medical management with tranexamic acid and oral and intrauterine progesterone for 2 years. She presented with right leg swelling and discoloration for 1 day, and a doppler ultrasound confirmed an extensive DVT. Limb saving procedure was taken up immediately with right femoral vein catheterization and venoplasty followed by heparin infusion and thrombolysis for total 48 hours. After 12 hours of stopping heparin drip total laparoscopic hysterectomy was done in view of continuous bleeding and to decrease pressure on iliac vessels. Post-operative heparin infusion started in 8 hours and patient was discharged in stable condition 48 hours post-surgery. Histopathological examination confirmed adenomyosis with weight of specimen being $1.5 \mathrm{~kg}$. Large uterine fibroids are a well-known cause of DVT and/or pulmonary embolism (PE). However, reports of large uterine adenomyosis causing DVT secondary to pelvic compression are scanty.
\end{abstract}

Keywords: Abnormal uterine bleeding, Adenomyosis, DVT, Thrombolysis

\section{INTRODUCTION}

Venous thromboembolic disease including DVT and PE is a major cause of morbidity and mortality worldwide. Reported incidence rate for DVT alone (without PE), range from 45 to 117 , per 100,000 person-years, respectively. ${ }^{1}$

In female patients with suspected DVT or PE, other causes, including obstetric and gynaecological reasons, should be considered. ${ }^{2}$ Mass effect from benign space occupying lesions, including large uterine fibroids, can result in venous stasis of the lower limbs, leading to venous TE. However, reports of large adenomyotic uterus causing DVT lower limb as a result of pelvic compression are scanty. Adenomyosis is not routinely considered as a risk factor for DVT among young patients and may present sans classical risk factors. ${ }^{4}$

Extensive adenomyosis carries a potential risk of infarction and thrombosis and exacerbates menorrhagia via activation of coagulation and fibrinolysis during menstruation. ${ }^{5}$ Hysterectomy in woman with a large adenomyotic uterus with undetected or untreated DVT carries a high risk of postoperative thromboembolism.

\section{CASE REPORT}

A 48-year lady presented to our emergency department in Oct 2018 complaining of sudden onset right lower-limb swelling and discoloration (Figure 1). She denied breathlessness or chest pain. Her menstrual history included progressively worsening heavy and painful 
menses for past 2 years. She had history of severe anemia secondary to menorrhagia with multiple blood transfusions and medical management with progesterone and tranexamic acid. She had history of levonorgestrelreleasing intrauterine system (LNG-IUS) insertion 18 months back which was expelled within a month. She had history of one previous lower (uterine) segment caesarean section (LSCS). She had no addictions, including smoking, alcoholism and was not on oral contraceptives.

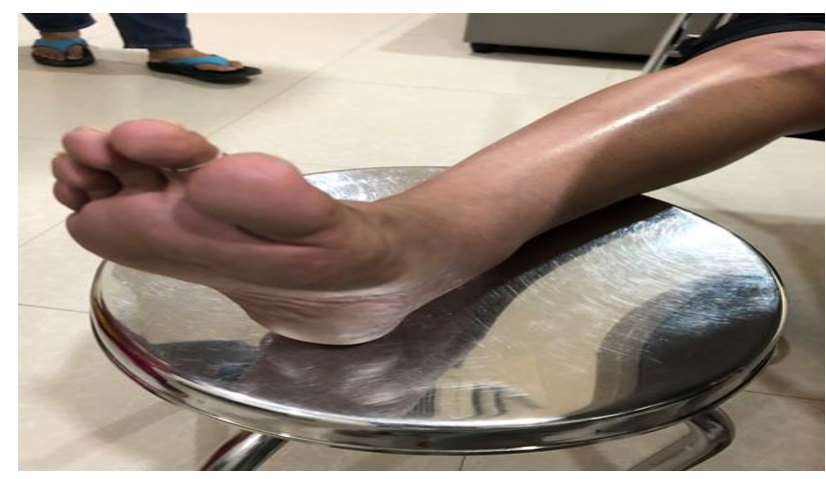

Figure 1: Right lower limb of oedema and discoloration.

On examination, she was found to have normal vitals except tachycardia. Per abdomen exam revealed a soft non tender abdomen with palpable uterus of 22 weeks size. On vaginal exam, the cervix appeared normal. There was a unilateral right calf swelling with severe tenderness. The right calf circumference measured $40 \mathrm{~cm}$ as compared to the left calf measuring $36 \mathrm{~cm}$. A positive Homan's sign was elicited in the right lower limb. The right femoral pulsations were difficult to palpate, otherwise the distal pulses were normal.

Her laboratory exam revealed haemoglobin of $9.1 \mathrm{gm} \%$ with a normal coagulation profile including protein $\mathrm{C}$, antithrombin III, factor V, homocysteine, lupus anticoagulants, and anticardiolipin levels. However, CA125 was elevated to $85 \mathrm{U} / \mathrm{ml}$. Lower limb venous doppler ultrasound confirmed an extensive DVT beginning in the right common iliac, extending to the external iliac and distally involving femoral vein (Figure 2). Pelvic ultrasound showed an enlarged uterus measuring $20.8 \times 10.4 \times 13.2 \mathrm{~cm}$ with diffuse adenomyosis (Figure 3).

A multidisciplinary team consisting of gynaecologists, interventional vascular radiologist, anaesthesiologist and internal medicine, was involved in the management.

Limb saving procedure was taken up immediately with right femoral vein catheterization and thrombolysis with heparin infusion. 24 hours later right femoral vein balloon venoplasty was done followed by heparin infusion and thrombolysis for total 48 hours. The indication of pelvic compression by the enlarged uterus and continued bleeding due to anticoagulants was suggestive of need of hysterectomy. With informed consent and anaesthetic fitness, the patient was planned up for laparoscopic hysterectomy under GA.

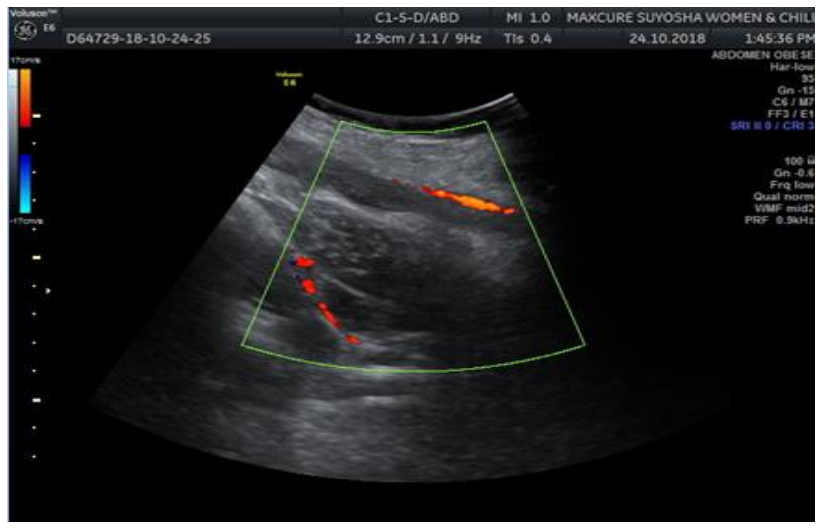

Figure 2: Doppler ultrasound of right lower limb of DVT in the right common iliac and femoral veins.

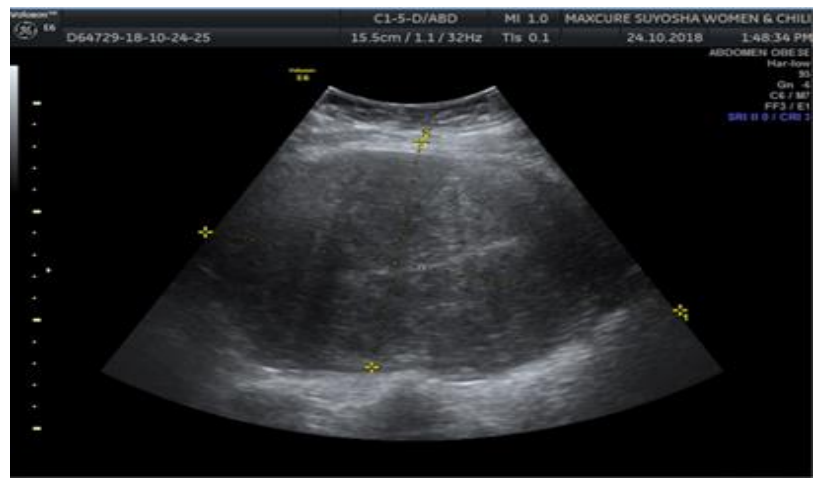

Figure 3: Pelvic ultrasound of an enlarged uterus with diffuse adenomyosis.

After 12 hours of stopping heparin drip total laparoscopic hysterectomy (TLH) with bilateral salpingectomy (BS) was done.

Abdomen entered with $5 \mathrm{~mm}$ right palmar port, $10 \mathrm{~mm}$ supraumbilical port and two accessory $5 \mathrm{~mm}$ left ipsilateral ports and pneumoperitoneum created. Uterus was enlarged to 20 weeks size with normal tubes and ovaries. TLH with BS done with ultrasonic scalpel (Figure 4). Specimen retrieved by vaginal morcellation (Figure 5). Vault closed with no. 1 vicryl hemostasis secured. Intraperitoneal drain kept in situ. She received transfusion with 2 units of packed red blood cells.

Post-operative patient stabilized in intensive care unit and heparin infusion started in 8 hours. The patient was discharged in stable condition 48 hours post-surgery on apixaban.

Histopathological examination revealed benign uterine changes with adenomyosis. Weight of specimen was 1.5 $\mathrm{kg}$. Follow up visits showed gradual recanalization of femoral veins on colour doppler study over a year. 


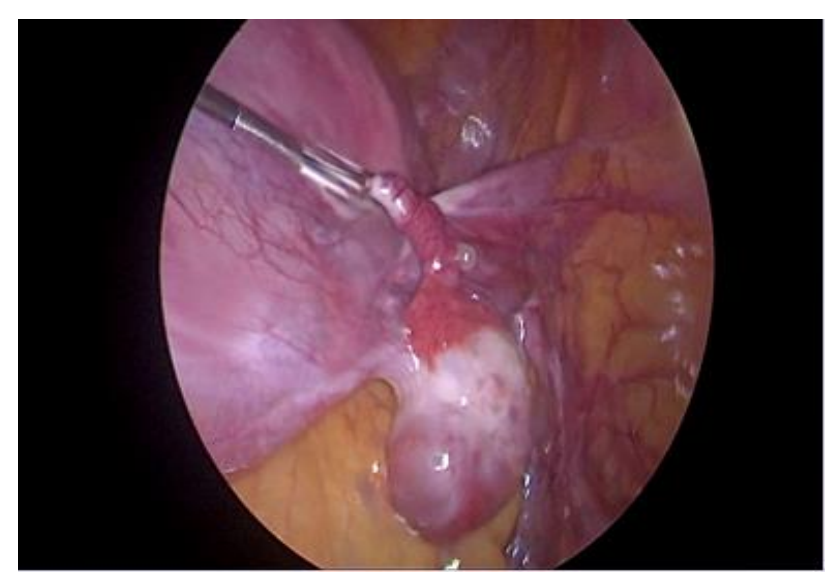

Figure 4: Laparoscopic view of enlarged uterus with right adnexa.

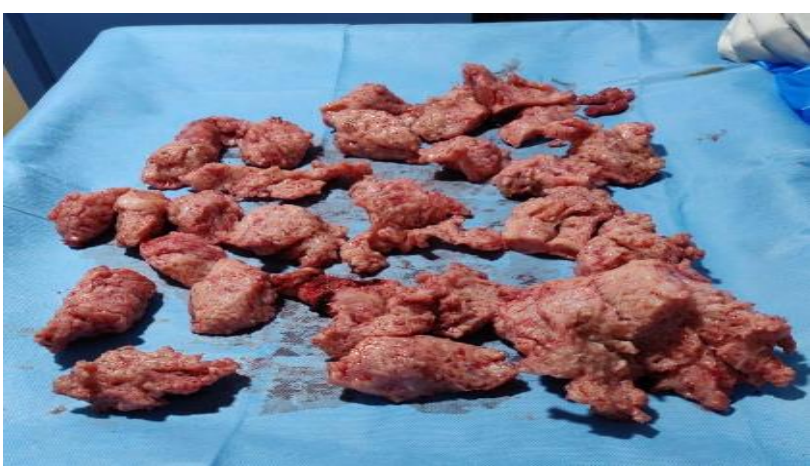

Figure 5: Specimen retrieved by vaginal morcellation.

\section{DISCUSSION}

DVT is the formation of blood clots in the deep venous system, most commonly the lower limbs. Acute DVT is characterized by sudden onset pain and swelling and is mostly occlusive whereas chronic DVT is symptomatic DVT that persists longer than 10 to 14 days. ${ }^{6}$ Risk factors for DVT and thromboembolism (TE) include increasing age, obesity, recent surgery, reduced mobility, trauma, cancer, use of estrogenic medications, migraine, illicit drug use, smoking, pregnancy and post postpartum period and deficiencies of blood coagulation agents like antithrombin III, protein $\mathrm{C}$, protein $\mathrm{S}$, and factor $\mathrm{V}$ Leiden mutation, hyperhomocysteinemia, antiphospholipid syndrome and inherited thrombophilias. ${ }^{3,6,7}$ The Virchow's triad-stasis, hypercoagulability, and endothelial disruption-is recognized as a risk factor for developing DVT. ${ }^{8}$

Adenomyosis is a benign lesion of myometrium with elevated CA125 level. It presents mostly in late reproductive years, with nonspecific symptoms like dysmenorrhea, menorrhagia, dyspareunia, chronic pelvic pain and infertility. ${ }^{5}$
Pressure effect from benign pelvic uterine masses, can result in venous stasis of the lower limbs, leading to venous TE. As such preventive anticoagulation is needed for larger uterine fibroid patients.?

In a study by Yamanaka et al, in the peripheral blood of eight patients it was found that activated fibrinolysis during menstruation may contribute to menorrhagia in patients with adenomyosis, Concentrations of thrombinantithrombin complex (TAT), soluble fibrin (SF), Ddimer(DD) and plasmin-alpha 2-plasmin inhibitor complex (PIC) were raised in patients with adenomyosis. TAT increased in 5 of the 6 patients with an adenomyotic uterus $\geq 100$ cubic centimeters. ${ }^{9}$

In a study by Yin et al, markedly increased CA125 and D-dimer levels were detected during menstruation, indicating activated coagulation system associated with CA125. ${ }^{4}$ Patients with adenomyosis may be at risk of hypercoagulability associated with increased CA125 level and menstruation-related activation of coagulation pathway leading to increased risk of thrombotic disorders. $^{4}$

Diagnosis is by D-dimer test, lower limb venous doppler USG, magnetic resonance imaging or computed tomography scan of the pelvis.

Given the high incidence of DVT in such patients, use of procoagulants (e.g., the oral contraceptive pill or tranexamic acid) should be best avoided for control of menorrhagia. $^{3}$

Treatment of DVT is anticoagulation by medications as low-molecular-weight heparin, warfarin, or other oral anticoagulants.

The main goal of treatment for women presenting with DVT/VTE secondary to large uterine masses is to tackle DVT first, followed by removal of uterus/mass. ${ }^{7}$ In the case studies reported, anti-coagulation associated with hysterectomy was the most commonly used treatment when DVT and PE developed secondary to uterine fibroids was diagnosed.?

The administration of an enzyme to break up clots is called thrombolysis. ${ }^{6}$ This reduces the risk of postthrombotic syndrome and the risk of leg ulcers. NICE recommends that thrombolysis is considered in those who have had symptoms for less than two weeks, are normally well, have a good life expectancy and a low risk of bleeding. ${ }^{6}$ A mechanical thrombectomy device can remove venous clots. Anticoagulation alone is suggested over thrombectomy. ${ }^{6}$

Alternative treatment for adenomyosis in patients not fit for surgery is gonadotropin-releasing hormone (GnRH) agonists/antagonists for decreasing the size of the uterus and decreasing pressure on deep veins. GnRH agonist therapy was useful as a uterus-conserving therapeutic 
option in patient who wished to retain fertility. ${ }^{10}$ Medical management of adenomyosis is contraindicated in women with a history of thromboembolism. ${ }^{8}$

Definitive treatment for symptomatic large uterus with pressure symptoms on deep pelvic veins is hysterectomy. Hysterectomy in such women can lead to pulmonary embolism due to release of occlusive effect and can have catastrophic effects. A pre hysterectomy inferior vena cava (IVC) filter would then reduce their risk of postoperative pulmonary embolism. ${ }^{6}$ IVC filters are also recommended for those with a contraindication to anticoagulant treatment. ${ }^{6}$

\section{CONCLUSION}

Large uterine masses can manifest with DVT even in the absence of the traditional risk factors. Physicians and obstetricians should be aware of a possibility of a large pelvic or intra-abdominal mass in a patient presenting with DVT or TE. Duplex ultrasound, done for screening of asymptomatic DVTs in women with large uterine masses, can be immensely helpful in avoiding and managing the associated peri and post-operative complications. To conclude, women with uterine mass more than $1000 \mathrm{gm}$ or more are at a significantly high risk of developing DVT. Thus, prevention of DVT requires avoidance of procoagulants in these women. Multidisciplinary approach with aggressive and prompt intervention in a diagnosed case is the key to lower both morbidity and mortality.

Funding: No funding sources Conflict of interest: None declared Ethical approval: Not required

\section{REFERENCES}

1. Heit_JA. Epidemiology of venous thromboembolism, Nat Rev Cardiol. 2015;12(8):464-74.

2. DH Lee, CW Kim, SE Kim. Deep Venous Thrombosis Caused by a Huge Uterine Myoma. Hong Kong J Emergency Med. 2017;19(5):361-3.

3. Ramanan S, Chapman-Wardy J, Watson R. Bleeding versus Clotting: A Complex Case of a Large Fibroid
Uterus Causing Menorrhagia and a DVT. Case Rep Obstetr Gynecol. 2016;2016:4.

4. Yin X, Wu J, Song S, Zhang B, Chen Y. Cerebral infarcts associated with adenomyosis: a rare risk factor for stroke in middle-aged women: a case series. BMC Neurol. 2018;18(213).

5. Onur I. Adenomyosis. Pathology Outlines.com website. Oct 8, 2019. Available at: http://www.pathologyoutlines.com/topic/uterusadeno myosis.html. Accessed on 27 July 2020.

6. Rao AS, Konig G, Leers SA, Cho J, Rhee RY, Makaroun MS, et al. (November 2009). "Pharmacomechanical thrombectomy for iliofemoral deep vein thrombosis: an alternative in patients with contraindications to thrombolysis". Journal of Vascular Surgery. 50 (5): 1092-8.

7. Ibrahim R, Dashkova I, Williams M, Khanna T, Kozikowski A, Dashkova A et al. Pulmonary embolism secondary to uterine fibroid: A case report of a rare presentation. J Phlebol Lymphol. 2018;11(1):13-5.

8. Garoufalis E. Lower-Extremity DVT Associated with a Large Uterine Leiomyoma, May 18, 2007. https://www.mdmag.com/journals/resident-andstaff/2006/2006-06/2006-06_01. Accessed on 27/7/2020.

9. Yamanaka A, Kimura F, Yoshida T, Kita N, Takahashi K, Kushima R et al. Dysfunctional coagulation and fibrinolysis systems due to adenomyosis is a possible cause of thrombosis and menorrhagia. Eu J Obst Gynecol Reprod Biol. 2016;4:99-103.

10. Akira S, Iwasaki N, Ichikawa M, Mine K, Kuwabara $\mathrm{Y}$, Takeshita $\mathrm{T}$, et al. Successful long-term management of adenomyosis associated with deep thrombosis by low-dose gonadotropin-releasing hormone agonist therapy, Clin Exp Obstet Gynecol. 2009;36(2):123-5.

Cite this article as: Anagani M, Agrawal P, Agrawal R. Acute right lower limb deep venous thrombosis due to pressure from large adenomyotic uteruslaparoscopic management. Int J Reprod Contracept Obstet Gynecol 2020;9:4749-52. 